\title{
Evaluation of the antitumor effects of PP242 in an LS174T cell-induced colon cancer xenograft model using comprehensive metabolomics and lipidomics
}

\author{
Md Mamunur Rashid ${ }^{1}$, Hyunbeom Lee $^{1}$, and Byung Hwa Jung ${ }^{1}$ \\ ${ }^{1} \mathrm{KIST}$
}

May 5, 2020

\begin{abstract}
BACKGROUND AND PURPOSE PP242, an inhibitor of mechanistic target of rapamycin (mTOR), displays potent anticancer effects against various types of cancer. However, the underlying metabolic mechanism associated with the PP242 effects is not clearly understood. In this study, comprehensive metabolomics and lipidomics investigations were performed in plasma and tumor tissue to reveal the metabolic mechanism of PP242 in an LS174T cell-induced colon cancer xenograft mouse model. EXPERIMENTAL APPROACH A colon cancer xenograft model was developed in BALB/c nude mice and then treated with PP242 for three weeks. After the final dose, blood, tumor, liver and kidney tissues were collected. Plasma and tumor samples were analyzed through untargeted metabolomics and lipidomics approaches using ultra-high-performance chromatographyOrbitrap-mass spectrometry (UHPLC-Orbitrap-MS). KEY RESULTS PP242 treatment reduces tumor size without any critical toxicities. According to results, metabolic changes due to the effects of PP242 were not significant in plasma. In contrast, metabolic changes in tumor tissues were very significant in the PP242-treated group compared to the xenograft control (XC) group, and revealed that energy and lipid metabolism were mainly altered by PP242 treatment like other cancer inhibitors. Additionally, it this study it was discovered that not only TCA cycle but also fatty acid $\beta$-oxidation ( $\beta$-FAO) for energy metabolism was inhibited and clear reduction in glycerophospholipid was observed. CONCLUSIONS AND IMPLICATIONS The findings of this study reveal new insights into the underlying anticancer mechanism of the dual mTOR inhibitor PP242, and could help further facilitate the understanding of the effects PP242 in the course of clinical application.
\end{abstract}

\section{Bullet Point Summary}

\section{What is already known}

PP242 is an ATP-competitive mTOR inhibitor that represents promising anticancer activity against various cancers.

\section{What this study adds}

- The antitumor activity of PP242 is mainly associated with energy and glycerophospholipid metabolism.

- Both the TCA cycle and fatty acid $\beta$-oxidation ( $\beta$-FAO) for energy metabolism were inhibited.

\section{Clinical significance}

This study can help to further understand the therapeutic effects of PP242 in clinical applications. 


\section{Introduction}

Colon cancer also known as colorectal cancer (CRC) or bowel cancer, is the fourth most common cancer and the third leading cause of cancer-related deaths among all cancers worldwide (Bray et al., 2018). The progression of CRC is associated with multiple genetic alterations, such as the activation of Ras, the overactivation of the phosphatidylinositol 3-kinase pathway, mutation of p53, and Wnt pathway dysregulation (Wang \& Zhang, 2014). In addition, emerging data have implicated frequent activation of mTOR with the progression of most cancers, including CRC, (Francipane \& Lagasse, 2013; Tian, Li, \& Zhang, 2019) which represents mTOR as a compelling therapeutic target for cancer treatment.

The mammalian or mechanistic target of rapamycin (mTOR) is a serine/threonine protein kinase that regulates many cellular functions, such as protein synthesis, cell proliferation, growth, survival, metabolism, autophagy and senescence, through two distinct multiprotein complexes named mTOR complex 1 (mTORC1) and mTOR complex 2 (mTORC2) (Johnson, Rabinovitch, \& Kaeberlein, 2013; Laplante \& Sabatini, 2012). mTOR regulates normal cellular functions through integrating signals from its upstream proteins PI3K and AKT (the PI3K/AKT/mTOR pathway). Dysregulation of this pathway leds to the development of several fatal diseases, including metabolic, cardiovascular, neurological, and immunological diseases and cancers (Tsang, Qi, Liu, \& Zheng, 2007). Since the mTOR pathway is dysregulated in many cancers, inhibition of mTOR represents a compelling therapeutic target for cancer treatment.

Depending on the inhibitory characteristics of mTOR complexes (mTORC1 and mTORC2), mTOR inhibitors are classified into two generations. Rapamycin and its analogs are considered first-generation mTOR inhibitors due to their ability to inhibit only mTORC1 activity (Mecca et al., 2018). Although prolonged exposure to rapamycin can also partially inhibit mTORC2, the inhibition is transient and not efficient for cancer treatment (Sarbassov et al., 2006). Therefore, efforts have been made to develop second-generation mTOR inhibitors that can efficiently inhibit both mTOR complexes. To date, numerous second-generation mTOR inhibitors have been developed to efficiently treat cancers. PP242 is an ATP-competitive second-generation inhibitor of mTOR that exerts its anticancer activity on numerous types of cancer. Previous studies have reported that PP242 can inhibit the proliferation, migration, invasiveness and stemness of glioblastoma cells (Mecca et al., 2018), induce apoptosis of acute myeloid leukemia and myeloid leukemia cells (Janes et al., 2010; Zeng et al., 2012), and suppress cell proliferation and migration of bladder and gastric cancer cells (Xing et al., 2014; Z. Zhang, Zhang, Kong, \& Gong, 2016). Furthermore, PP242 also reduced the growth, proliferation and survival of colon cancer cells, and in combination with cetuximab, PP242 shows synergistic activity towards colorectal carcinoma (Blaser et al., 2012; Cheng et al., 2015). However, the underlying mechanism of the effects of PP242 on the basis of the metabolome is still not clearly understood.

Metabolomics, the comprehensive study of small molecule metabolites, appears to be a robust tool to assess diverse biological samples, including cells, biofluids and tissues, in response to biological stimuli, such as disease and drugs (Qiu et al., 2009; Zheng et al., 2017). Lipidomics, which is a subset of metabolomics, mainly contributes in the evaluation of various lipid species in complex biological samples (Murfitt et al., 2018). Combined metabolomics and lipidomics approaches have been widely applied and used assess the pathophysiological pathways related to disease progression, identify biomarkers and monitor the underlying mechanism of drug effects and toxicity (Rivera- Velez et al., 2019).

In this study, we examined the effects of the second-generation mTOR inhibitor PP242 in LS174T cell-induced colon cancer xenograft model mice in order to reveal the underlying mechanism of PP242. Comprehensive metabolomics and lipidomics profiling approaches using mouse plasma and tumor tissues were applied to investigate PP242 effects using an UHPLC-Orbitrap-MS system.

\section{Materials and methods}

\section{Materials}

The mTOR inhibitor PP242 (torkinib, purity 97.75\%) was purchased form MedChemExpress (NJ, USA). Internal standards (ISs), reserpine, and 1-Hexadecanoyl ( $\left.\mathrm{d}_{31}\right)$-2-(9Z-octadecenoyl)-sn-glycero-3-phosphocholine 
(PC(16:0/18:1)- $\left.\mathrm{d}_{31}\right)$ and arachidonic acid- $\mathrm{d}_{8}$ were purchased from Sigma-Aldrich Chemical Co. (St. Louis, MO. USA) and Avanti Polar Lipids (Alabaster, AL, USA), respectively. Formic acid, ammonium acetate and $10 \%$ neutral buffered formalin solution were purchased from the Sigma-Aldrich Chemical Co. (St. Louis, MO, USA). Ultrapure water (18.2 M $\Omega . c m$ ) was generated using a Milli-Q apparatus from Millipore (Milford, MA, USA). All other chemicals were of highest analytical grades and HPLC grade organic solvents were used for the preparation of mobile phases and supplied by Burdick \& Jackson (SK chemicals, Ulsan, Korea).

\section{Cell lines}

The human colon cancer cell line LS174T was purchased from American Type Culture Collection (ATCC; Manassas, VA, USA) and maintained in Dulbecco's modified eagle's medium (DMEM) supplemented with $10 \%$ fetal bovine serum (FBS) and $1 \%$ penicillin-streptomycin in a humidified $5 \% \mathrm{CO}_{2}$ and $37{ }^{\circ} \mathrm{C}$ incubator.

\section{Development of xenograft model}

All of the experimental procedures were permitted by the Institutional Animal Ethics Committee of the Korea Institute of Science and Technology (KIST), Korea. Fifteen male BALB/c nude mice aged 6 weeks weighing 23-27 g were purchased from Nara Controls Inc. (Seoul, Korea). The mice were then housed for one week to acclimate to the ambient environment, and during the acclimation period, the temperature $(25$ $\left.\pm 2{ }^{\circ} \mathrm{C}\right)$, the humidity $(50-60 \%)$ and a $12 \mathrm{~h}$ light/dark cycle $(8: 00 \mathrm{am}-8: 00 \mathrm{pm})$ were maintained. The mice were initially divided into two groups: the normal control group $(\mathrm{NC} ; \mathrm{n}=5)$ and the tumor xenograft group $(n=10)$. After one week, one million LS174T cells were subcutaneously injected into the right flank of the nude mice for xenograft model development, and blank media was injected into the normal control group following the same xenograft model procedure. Once the tumor size reached approximately 150-200 $\mathrm{mm}^{3}$, the xenograft group was further divided into two groups: a xenograft control group (XC; treated with vehicle, $\mathrm{n}=5$ ), and a PP242-treated group (treated with PP242; $\mathrm{n}=5$ ). PP242 solution (formulated with $2 \%$ ethanol, 20\% PEG400 and 1\% Tween 80 in distilled water) was administered orally to the xenograft mice at a dose of $60 \mathrm{mg} \cdot \mathrm{kg}^{-1}$. day ${ }^{-1}$ for 21 continuous days. The same volume of vehicle was orally administered to the $\mathrm{NC}$ and $\mathrm{XC}$ group mice for the same duration. Measurements of tumor volume in the right flank region and body weights were carried out in two day intervals. The tumor size was measured using a digital caliper and calculated with the formula:

Volume $=$ weight $^{2} \times \frac{\text { Length }}{2}$.

After 21 days of treatment, the mice were anaesthetized, and blood was collected through cardiac puncture. Liver, kidney and tumor tissues were collected and immediately snap frozen in liquid nitrogen. Plasma was then obtained from the blood samples upon centrifugation. All the samples were stored at $-80{ }^{\circ} \mathrm{C}$ until further analysis.

\section{Serum biochemical parameters and histological analysis}

In order to investigate the toxicity-related effects of PP242, serum biochemical parameters were measured. Aspartate aminotransferase (AST), alanine aminotransferase (ALT) and total bilirubin were measured to determine liver health; blood urea nitrogen (BUN) and creatinine (CRE) were measured to evaluate kidney health; and low-density lipoprotein (LDL) and high-density lipoprotein (HDL) were measured to evaluate heart health.

Histopathological examination was conducted with hepatic and renal tissues obtained from the trial fixed in primary $10 \%$ neutral formalin solution. The left lobe of the liver and kidneys were cut to an appropriate size and thickness and fixed in the secondary formalin solution. The cut tissue was embedded in paraffin through a general tissue treatment process. Paraffin-formatted tissues were then cut to a thickness of $3 \mu \mathrm{m}$ and subjected to hematoxylin and eosin staining, and histopathological examination was performed under an optical microscope (Olympus CX41, Japan). The findings were recorded, depending on the degree as normal, minimal $(1+)$, mild $(2+)$, moderate $(3+)$ or severe (severe, $4+)$ grading.

Sample preparation for metabolomics and lipidomics study 


\section{Plasma}

For the metabolomics study, plasma samples were processed through a simple protein precipitation technique using $150 \mu \mathrm{L}$ of ice-cold methanol, with plasma to methanol ratio was of 1:3. After the addition of methanol, the mixture was then vortexed and centrifuged at $14,000 \mathrm{rpm}$ for $10 \mathrm{~min}$ at $4{ }^{\circ} \mathrm{C}$. The resulting supernatant was transferred to a new tube and diluted with water containing IS (reserpine; $4 \mu \mathrm{g} . \mathrm{mL}^{-1}$ ) at a ratio of $2: 1$. Finally, $5 \mu \mathrm{L}$ of sample was injected into the UHPLC-Orbitrap-MS system after slight vortexing and spinning down. A quality control (QC) sample was made by gathering identical volumes of plasma samples and then diluting them with water containing IS (reserpine; $4 \mu \mathrm{g} \cdot \mathrm{mL}^{-1}$ ) using the same ratio mentioned above. QC samples were used to evaluate the repeatability and robustness of the instrumental system and were analyzed before starting the sequence for column conditioning and after every ten samples in the analytical batch. Additionally, test mixtures containing a few commercially available validated standards were run at the beginning, middle and end of the analytical batch. The test mixture contained the following compounds: caffeine $\left(0.5 \mu \mathrm{g} . \mathrm{mL}^{-1}\right)$ and acetaminophen $\left(0.5 \mu \mathrm{g} . \mathrm{mL}^{-1}\right)$ for positive mode and glycocholic acid $\left(0.5 \mu \mathrm{g} . \mathrm{mL}^{-1}\right)$ and hippuric acid $\left(0.5 \mu \mathrm{g} \cdot \mathrm{mL}^{-1}\right)$ for negative mode.

For the lipidomics study, $25 \mu \mathrm{L}$ of PC (16:0/18:1)- $\mathrm{d}_{31}\left(4 \mu \mathrm{g} \cdot \mathrm{mL}^{-1}\right.$; internal standard for positive mode), 25 $\mu \mathrm{L}$ of arachidonic acid- $\mathrm{d}_{8}\left(4 \mu \mathrm{g} \cdot \mathrm{mL}^{-1}\right.$; internal standard for negative mode), and $50 \mu \mathrm{L}$ of $0.1 \mathrm{M} \mathrm{NaCl}$ were added to $50 \mu \mathrm{L}$ of the plasma samples. Lipid extraction was then performed by the addition of $250 \mu \mathrm{L}$ of ice-cold chloroform/methanol $(1: 2 ; \mathrm{v} / \mathrm{v})$ to the plasma mixture. The mixture was then vortexed for 1 min, kept at room temperature for $1 \mathrm{~h}$, and centrifuged at $14,000 \mathrm{rpm}$ for $10 \mathrm{~min}$ at $4{ }^{\circ} \mathrm{C}$. The clear supernatant was transferred to a new tube and evaporated to dryness under a nitrogen stream at $37^{\circ} \mathrm{C}$. Finally, the dried residue was reconstituted using $60 \mu \mathrm{L}$ of ice-cold chloroform/methanol $(1: 1 ; \mathrm{v} / \mathrm{v})$ before being injected into the instrument for analysis. A QC sample was also prepared by gathering identical volumes from each sample after reconstitution in order to assess the repeatability and robustness of the instrument. All QC samples were run following a sequence similar to that of the metabolomics analysis.

\section{Tumor tissue}

The snap-frozen liver and kidney tissues were lyophilized and homogenized into powder. Approximately 10 mg of homogenized tissue was transferred to a $2 \mathrm{~mL}$ Eppendorf tube, and $400 \mu \mathrm{L}$ of methanol was added. The mixture was then sonicated for $2 \mathrm{~min}$ and $1 \mathrm{~mL}$ of MTBE (methyl tert-butyl ether) was added followed by $1 \mathrm{~h}$ of shaking in a shaking water bath at room temperature. Separation was induced by the addition of $250 \mu \mathrm{L}$ of water followed by room temperature incubation for $10 \mathrm{~min}$. After 15 min of centrifugation at $14,000 \mathrm{rpm}$ at $4{ }^{\circ} \mathrm{C}$, the supernatant was separated (upper organic layer and lower polar layer). For the metabolomics study, $200 \mu \mathrm{L}$ of supernatant was taken and evaporated to dryness under a nitrogen stream at $37^{\circ} \mathrm{C}$. The dried residue was then reconstituted in $100 \mu \mathrm{L}$ of $80 \%$ methanol, and $50 \mu \mathrm{L}$ was loaded into the UHPLC-Orbitrap-MS system for analysis. For the tissue lipidomics study, the same volume of supernatant was taken, processed following the same method and finally reconstituted in $100 \mu \mathrm{L}$ of chloroform/methanol $(2: 1)$. In order to normalize tissue metabolomics and lipidomics data, the protein concentration of each sample was quantified. Each sample was diluted with distilled water, and the protein concentration was measured by Nano-MD (SINCO, Korea) using $10 \mu \mathrm{L}$ of sample. For both the tissue metabolomics and lipidomics analyses, QC samples were made by taking identical volumes from each sample and running the samples following a sequence similar to that of plasma analysis.

\section{Instrumental conditions}

Instrumental analysis was carried out using an Ultimate 3000 UHPLC system coupled to an LTQ Orbitrap Velos Pro mass spectrometer system (Thermo Fisher Scientific, San Jose, CA, USA) with a heated electrospray ionization (HESI) source. The same instrumental conditions and methods were used for the plasma and tumor tissue metabolomics and lipidomics analyses.

For the metabolomics analysis, an ACQUITY UPLC BEH C18 column $(2.1 \times 100 \mathrm{~mm}, 1.7 \mu \mathrm{m}$, Waters, Milford, MA, USA) was used for the chromatographic separation by maintaining the autosampler and column oven temperature at $4{ }^{\circ} \mathrm{C}$ and $50{ }^{\circ} \mathrm{C}$, respectively. Formic acid $(0.1 \%)$ in distilled water (v/v, mobile phase 
A) and methanol (v/v, mobile phase B) was used as the mobile phase and eluted at a flow rate of 0.4 $\mathrm{mL} \cdot \mathrm{min}^{-1}$ throughout the entire analysis. The elution gradient was regulated as follows: the elution started with $100 \% \mathrm{~A}$ and was maintained for $1 \mathrm{~min}$, then gradually decreased to $80 \%$ A over next 4 min, and a linear decrease of mobile phase A was made from $80 \%$ to $30 \%$ from 4 to $10 \mathrm{~min}$. Mobile phase A was then decreased to $0 \%$ at $14 \mathrm{~min}$ followed by a rapid increase to the initial conditions for re-equilibration at the initial conditions for 2 min.

For the lipidomics analysis, chromatographic separations were performed using an ACE Excel 2 Super C18 column $(2.1 \times 100 \mathrm{~mm}, 1.7 \mu \mathrm{m}$, Advanced Chromatography Technologies Ltd., Aberdeen, Scotland, UK), and the autosampler and column oven temperature were maintained at $4{ }^{\circ} \mathrm{C}$ and $50{ }^{\circ} \mathrm{C}$, respectively. The mobile phase was composed of $10 \mathrm{mM}$ ammonium acetate in either $40 \%$ acetonitrile $(\mathrm{v} / \mathrm{v}$, mobile phase A) or acetonitrile: isopropanol (10:90, v/v, mobile phase B) and eluted at the same flow rate as that of the metabolomics study. The elution gradient was initiated using $60 \% \mathrm{~A}$ that was maintained for $1 \mathrm{~min}$, and from 1 to $3 \mathrm{~min}$, the concentration of A decreased to $35 \%$. Over the next $2 \mathrm{~min}$, mobile phase A decreased to $15 \%$; then, over the next $4 \mathrm{~min}$, it further decreased to $0 \%$, and this condition was maintained for 3 min. Finally, mobile phase A was rapidly increased to reach the initial conditions for $0.5 \min (60 \% \mathrm{~A})$ and re-equilibrated for $3.5 \mathrm{~min}$. The injection volume was $5 \mu \mathrm{L}$ for all analyses.

Mass spectrometric (MS) conditions were the same for the metabolomics and lipidomics analyses of the plasma and tumor tissue samples, respectively. MS detection was operated in both positive and negative modes using a full scan ranging from $\mathrm{m} / z 50$ to 1600, and data were acquired in centroid mode at a resolution of 60,000. High-energy collision dissociation mode was employed for the dissociation of metabolites using a normalized collision energy of $35 \%$ with an isolation width of $1 \mathrm{~m} / \mathrm{z}$ and an activation time of $10 \mathrm{~ms}$. The detailed MS parameters were as follows: heater temperature, $40{ }^{\circ} \mathrm{C}$; sheath gas flow rate, 45 arb; auxiliary gas flow rate, $10 \mathrm{arb}$; spray voltage, $4 \mathrm{kV}$; capillary temperature, $320{ }^{\circ} \mathrm{C}$; and S-lens RF level, $61 \%$. For both the sheath gas and auxiliary gas nitrogen was used.

\section{Data processing and statistical analysis}

After the data acquisition using LTQ-Orbitrap, the raw data were imported and processed (peak alignment, detection and identification) using the Compound Discoverer 2.1 software system. The retention time and $\mathrm{m} / \mathrm{z}$ data for each peak were determined by the software. For plasma metabolomics and lipidomics analyses, all data were normalized using the peak areas of internal standards. For metabolomics and lipidomics profiling of tumor tissue samples, all data were normalized to the total protein concentrations. All multivariate data analyses were performed using SIMCA version 14.1 software (Umetrics, Inc., Ume, Sweden) system and MetaboAnalyst 4.0 (https://www.metaboanalyst.ca/). Multivariate data analyses and partial least squaresdiscriminant analysis (PLS-DA) were used to evaluate the differential metabolites between groups in the plasma and tumor samples, and performed with Pareto scaling. The components with a variable importance in the projection (VIP) value exceeding 1.0 were selected as potential compounds that contributed remarkably to the clustering and showed differences between the groups. Student's $t$-test was used to evaluate the statistical significance of each group of metabolic changes and considered significant when the value was less than 0.05 .

Putative identification and searching was carried out based on the mass adducts $\left([\mathrm{M}+\mathrm{H}]^{+},[\mathrm{M}+\mathrm{Na}]^{+},[\mathrm{M}-\right.$ $\mathrm{H}]^{-}$, etc), mass fragment $\left(\mathrm{MS}^{2}\right)$ ions and retention time. The accuracy tolerance window of the mass was set to $10 \mathrm{ppm}$ while searching the metabolites. The metabolites were initially searched and determined from online databases such as METLIN (https://metlin.scripps.edu/), HMDB (http://www.hmdb.ca/), and KEGG (http://www.genome.jp/kegg) utilizing the detected $\mathrm{m} / \mathrm{z}$ value and mass fragmentation patterns. Later, the identified metabolites were further crosschecked with the commercially available standards for the confirmation.

\section{Results}

Physical observations 
The body weights of the mice in each group (NC, XC, and PP242-treated) were measured once in every two days and compared (Fig. 1). Compared to the NC group, the body weights of the XC group mice decreased, but the changes were not significant. In contrast, the body weights of the PP242-treated mice decreased significantly and quickly in the first couple of days after treatment compared to the NC group, and later, the body weights gradually recovered. However, no significant change in body weight was observed between the XC and PP242-treated groups, indicating that the change in tumor size and drug treatment did not seriously affect body weight within 21 days.

Tumor-bearing mice were treated with vehicle and/or PP242 over the course of 21 days, and the size of the tumor in the right flank region was measured at two day intervals using digital caliper in the LS174T xenograft groups (XC and PP242-treated) in order to evaluate the drug therapeutic effects in vivo. After two weeks of PP242 treatment, a very significant difference in the tumor volumes was observed $(\mathrm{p}<0.001)$ before the mice were sacrificed (Fig. $2 \mathrm{~A}$ and $2 \mathrm{~B}$ ). The tumors were separated after sacrifice and weighed, which further displayed the significant decrease in tumor weight for the PP242 treatment compared to the $\mathrm{XC}$ group mice (Fig. 2C).

\section{Serum biochemical parameters and histopathological tests}

The results of the serum biochemical parameters test are shown in Fig. 3. All the measured serum biochemical parameters related to liver toxicity were in the normal range for all groups. No significant abnormalities were observed in the kidney toxicity biomarkers CRE and BUN either. BUN was significantly reduced only in the PP242-treated group compared to the NC group, but all were in the normal range. Alterations in cholesterol levels were observed, where LDL levels were reduced and HDL levels were increased after three weeks of PP242 treatment.

Histological examination of the liver showed no abnormal findings in all subjects tested in the $\mathrm{NC}$, XC and PP242-treated groups (Supplementary Table 1, Supplementary Fig. 1). In the histologic examination of the kidneys, no abnormal lesions were observed in any group except for cortical renal tubule hypertrophy that was observed in one-fifth of the PP242-treated group (Supplementary Table 1). However, it was thought that there was no kidney toxicity since the degree of hypertrophy was very minimal and the serum biochemical parameters for kidney function were normal (Supplementary Fig. 1).

Taken together, no unusual toxicological changes were observed in the liver or kidney when PP242 was administered according to the given experimental design.

\section{Identification of altered endogenous metabolites}

In order to evaluate the underlying mechanism of action of the dual mTOR inhibitor PP242, the LS174T xenograft mouse model was treated with PP242 for three weeks, and plasma and tumor samples were analyzed via metabolomics and lipidomics approaches. The representative base peak intensity (BPI) chromatograms of the plasma and tumor tissues for metabolomics and lipidomics studies in both positive and negative ion modes are displayed in Supplementary Figs. 2 and 3. To clearly visualize the metabolic differences among the NC, XC, and PP242-treated groups in plasma and the XC and PP242-treated groups in tumor tissues, multivariate statistical analysis was used to analyze the processed mass spectrometric data. PLS-DA score plots of plasma and tumor tissue metabolomics and lipidomics are shown in Figs. 4 and 5. In the plasma metabolomics and lipidomics analyses, the xenograft groups (XC and PP242-treated) were clearly separated from the NC group, indicating metabolic differences due to tumor formation, but the XC and PP242-treated groups slightly overlapped (specifically in positive metabolomics; Fig. 4). In addition, the PLS-DA score plots of tumor tissues displayed a clear separation between the XC and PP242-treated groups, which clearly indicates that metabolism in tissue was altered due to the inhibition of mTOR by PP242 and that these metabolic changes were clearer in tissue than in plasma. The $\mathrm{R}^{2}$ and $\mathrm{Q}^{2}$ values for plasma metabolomics and lipidomics were in the ranges of 0.43 to 0.61 and 0.1 to 0.20 , respectively, whereas those for tumor tissue were in the ranges of 0.82 to 0.99 and 0.04 to 0.87 , respectively. The overall $\mathrm{R}^{2}$ and $\mathrm{Q}^{2}$ values showed that the model was reliable and had good predictability. The obtained low $\mathrm{Q}^{2}$ value could be because of the partial overlap of the XC and PP242 groups. 
In the metabolomics and lipidomics profiling of plasma, comparisons were carried out among the $\mathrm{NC}, \mathrm{XC}$ and PP242 groups. The xenograft groups (XC and PP242-treated) were compared with the NC group to investigate the metabolic differences that occurred due to tumor formation and how much was recovered after PP242 treatment. Initially, a total of 49 significantly altered metabolites were identified. Of these, 22 metabolites were finally selected and used for further analyses by considering a VIP value $>1.0$ and a $\mathrm{p}$ value (obtained by Student's $t$ test $)<0.05$. Detailed information on the identified metabolites in plasma is listed in Table 1. A heat map was also used to visualize the change pattern and is displayed in Fig. 6 . The identified metabolites were mostly limited to glycerophospholipids, fatty acids and a few organic compounds. Compared to the NC group, the overall change patterns in metabolites in both xenograft groups were similar. Interestingly, none of the identified metabolites were significantly altered in the XC group compared with the PP242-treated group, and there was no consistency in the alteration pattern of all metabolite classes. Consequently, a potential effect of PP242 was not observed in plasma. However, when we observed the tumor volume and weight (Fig. 2), the changes were significant after treatment with PP242, indicating an obvious therapeutic effect of PP242. Hence, in order to investigate the underlying meaning of the PP242 effect in tumor size reduction, we further analyzed tumor tissues.

In tumor tissue, compared to the XC group, a total of 93 significantly altered metabolites were identified in the PP242-treated group via metabolomics and lipidomics analyses. Considering a VIP $>1.0$ and a $\mathrm{p}$ value $<0.05,59$ metabolites were ultimately selected and used for further analysis (Table 2). A wide range of metabolites, including a few other organic compounds, fatty acyls, glycerophospholipids, glycerolipids and sphingolipids, were significantly altered after PP242 treatment. The metabolites related to energy production (lactate, aspartic acid, fatty acids and carnitines) and cell growth and proliferation (glycerophospholipids) were mainly affected. We observed that the levels of all carnitines were decreased, and fatty acids, which are the precursors of various lipid molecules and are involved in energy generation, were increased due to the inhibition of mTOR by PP242. Other significantly altered metabolite classes were also identified, including amino acids (L-methionine), carboxylic acids and derivatives (creatine and L-aspartic acid), hydroxyl acids and derivatives (L-lactic acid), purine nucleotides (inosine), pyrimidine nucleotides (uridine 5'-monophosphate) and phenylpropanoic acids (phenyllactic acid). All glycerophospholipids, including phosphatidylcholine (PC), phosphatidylethanolamine (PE), phosphatidylinositol (PI) and phosphatidylserine (PS), showed significant downregulation in the PP242-treated group compared to the XC group. However, only one identified LysoPC (LysoPC(18:2)) was significantly altered and showed upregulation after mTOR inhibition. The level of glycerolipid DG(36:2) was significantly upregulated, and the sphingolipid $\operatorname{SM}(34: 1)$ level was significantly downregulated. A heat map is displayed in Fig. 7 to display the direct variation of each differential metabolite.

\section{Discussions}

Mammalian target of rapamycin (mTOR) is frequently activated and overexpressed in a variety of cancers, including colon cancer. Hence, inhibition of mTOR is one of the crucial therapeutic steps in the course of cancer treatment. PP242 is an ATP-competitive inhibitor of mTOR, and the activity of PP242 in the inhibition of colon cancer growth in vitro and in vivo has been previously reported (Blaser et al., 2012; Gulhati et al., 2009; Roulin, Cerantola, Dormond-Meuwly, Demartines, \& Dormond, 2010; Y.J. Zhang et al., 2009). However, the underlying metabolic mechanism behind the effects of PP242 is still not clear. In the present study, using comprehensive metabolomics and lipidomics approaches, we identified that the antitumor effects of PP242 are associated with the inhibition of energy metabolism pathways, including glycolysis, the TCA cycle, fatty acid $\beta$-oxidation ( $\beta$-FAO) and glycerophospholipid metabolism, in an LS174T cell-induced colon cancer xenograft mouse model.

According to the serum biochemistry and tissue histopathology test results, no unusual toxicological changes were observed in the liver and kidney when PP242 was administered at a daily dose of $60 \mathrm{mg} \cdot \mathrm{kg}^{-1}$. day ${ }^{-1}$ for 21 days, and these data were supported by previously published data (Tanaka et al., 2015). Surprisingly, PP242 reduced LDL levels and increased the HDL levels in mice with colon cancer. This means that the change in lipid metabolism by PP242 treatment could also affect cholesterol metabolism. 
In plasma, in order to disclose the therapeutic effects of PP242, a comparison was carried out among the three experimental groups (NC, XC, and PP242-treated). A total of 22 metabolites were identified in plasma, which were significantly altered in either the XC or PP242-treated group. However, no metabolites were significantly altered between the XC and PP242-treated groups (Table 1). We observed that despite having the visible effect of PP242 in reducing tumor size (Fig. 2), changes in the plasma metabolome were not consequential to conclude the underlying mechanism. Moreover, changes in the metabolites were inconsistent, where the XC and PP242-treated groups displayed identical patterns of change among the metabolites compared to the NC group. Therefore, we analyzed the tumor tissues to further uncover PP242 activity at the metabolome level.

The comprehensive metabolomics and lipidomics profiling of tumor tissues provided a better understanding and delineation of the underlying mechanism of PP242 compared to that provided by the plasma analysis, and the major perturbed metabolic pathways were mainly related to energy metabolism (glycolysis, the TCA cycle, and $\beta$-oxidation of mitochondrial fatty acids) and glycerophospholipid metabolism. In tumors, due to the daily dose of PP242 for three weeks, 59 metabolites, including a few organic metabolites, fatty acids, glycerophospholipids, glycerolipids and sphingolipids, were significantly altered (Table 2).

In order to maintain growth and survival, most cancer cells rely highly on glycolysis to fulfill the elevated demand for nutrients and energy, which finally leads to the elevation of lactic acid levels (Warburg, 1956; Hay, 2016; Mason \& Rathmell, 2011). This elevation in lactic acid levels causes acidosis in the extracellular tumor microenvironment to maintain $\mathrm{pH}$ homeostasis and supports the migration and invasion of cancer cells (de la Cruz-Lopez, Castro-Munoz, Reyes-Hernandez, Garcia-Carranca, \& Manzo-Merino, 2019; Alfarouk et al., 2014; Goetze, Walenta, Ksiazkiewicz, Kunz-Schughart, \& Muller-Klieser, 2011). In the present study, after PP242 treatment, the lactic acid level was significantly decreased in the tumors, indicating inhibition of glycolysis and thereby inhibiting cancer growth and invasion. We also observed a significant reduction in the level of the TCA cycle intermediate aspartic acid after PP242 treatment. Aspartic acid is the degradation metabolite of the TCA cycle, which is produced from the glutaminolysis pathway. In the glutaminolysis pathway, the production of $\alpha$-ketoglutarate from glutamine via glutamic acid is another main metabolic pathway for tumor growth and survival that replenishes TCA cycle energy demand by acting as a carbon source (Jin, Alesi, \& Kang, 2016; Tanaka et al., 2015). Thus, the decrease in the aspartic acid level reflects the alteration of metabolites that may reduce the tumor growth of colon cancer xenograft mice by inhibiting the energy supply to the TCA cycle.

In addition to glycolysis, to meet the increased energy demand, cancer cells carry out other metabolic strategies, such as $\beta$-FAO, to produce more energy to support cancer cell growth and survival. Carnitine plays a major role in transporting long-chain fatty acids inside the inner membrane of mitochondria, which facilitates $\beta$-FAO to generate and supply acetyl-CoA to the TCA cycle for energy production (Baci et al., 2018; Melone et al., 2018; Qu, Zeng, Liu, Wang \& Deng, 2016). Previous studies have reported that higher blood carnitine levels denote higher energy and functioning of cells, which is the main requirement of cancer cells. Furthermore, increased levels of carnitines were also reported in breast cancer and chronic lymphocytic leukemia (Armitage \& Southam, 2016; Cala et al., 2018). According to our results, as the level of carnitines, including L-carnitine, L-acetylcarnitine, and L-palmitoylcarnitine, decreased after mTOR inhibition by PP242, the transport of fatty acids also decreased, resulting in an increase in fatty acid levels. Hence, it could be speculated that inhibition of mTOR signaling by PP242 acts by blocking $\beta$-FAO in cancer cells by reducing carnitine levels.

Treatment with PP242 also significantly reduced the glycerophospholipid (PC, PE, and PI) levels, except lysophosphatidylcholine (LysoPC), where only one LysoPC (LysoPC18:2) was significantly affected and whose level increased. Phosphatidylcholine (PC) is a fundamental element of the cell membrane and plays a pivotal role in the structure and function of cell membranes (Furse \& de Kroon, 2015; Gibellini \& Smith, 2010). Upregulation of PC has been observed in numerous cancers, including colon cancer, and is considered one of the hallmarks of cancer growth and progression (Cheng, Bhujwalla, \& Glunde, 2016; Jones et al., 2019; Kurabe et al., 2013). In this experiment, after PP242 treatment, the level of PC was significantly 
downregulated, suggesting that PP242 is able to inhibit cancer growth and progression. One LysoPC was significantly increased when the mice were exposed to PP242. LysoPC is normally generated from PC through the catalysis of phospholipase $\mathrm{A}_{2}\left(\mathrm{PLA}_{2}\right)$, and few studies have reported that the upregulation of LysoPC can be associated with the induction of apoptosis (Law et al., 2019; M. Takahashi et al., 2002). Therefore, according to our results, the increase in LysoPC levels could be due to the apoptotic effects of PP242. Besides, LysoPC could also increase due to the inhibition of its conversion from PC, which also decreases the level of PC (Gao et al., 2016).

Phosphatidylethanolamine (PE) is another vital element of phospholipids, and in mammalian cells, PE accounts for almost 15-25\% of the total lipid content and is also associated with a vast number of physiological cellular processes (Lu et al., 2019; Patel \& Witt, 2017; Tan et al., 2017). In the normal cellular state, the existence of PE is only found in the inner leaflet of the cell membrane. However, upregulation of PE on the outer surface of cancer cells has been previously reported (Stafford \& Thorpe, 2011; Tan et al., 2017). PE was significantly downregulated after PP242 treatment in our study, suggesting the potential of PP242 in treating cancer by decreasing PE levels.

We also observed a significant reduction in all phosphatidylinositol (PI) species after exposure to PP242 for three weeks. PI is another important phospholipid class, accounting for approximately $5.6 \%$ of total lipids, mainly exists in the inner leaflet of the cell membrane, and plays a role in regulating cell survival, signaling and membrane trafficking (Duan, 2016; Falkenburger, Jensen, Dickson, Suh, \& Hille, 2010). PI overexpression is also associated with cancer progression, and thus, a decrease in PI levels has shown cancer growth suppression against various cancers (Baba et al., 2001; Kim, Jin, Bae, \& Choi, 2019; Imoto et al., 1998). This evidence of PI level reduction supports our results and indicates the potent antitumor activity of PP242.

Among the anionic phospholipids, phosphatidylserine (PS) is the most abundant and is located in the plasma membrane's inner leaflet of most mammalian cells. It has also been reported that cancer cells possess elevated levels of PS on their external surface (Davis et al., 2019; Vallbhapurapu et al., 2015). Hence, the decrease in PS levels, could suggest the suppression of cancer growth by the effects of PP242 treatment. Diacylglycerol (DG) and sphingomyelin (SM) were significantly increased and decreased, respectively. In cellular signaling and lipid metabolism, DG plays a very important role as an intermediate (Cala et al., 2018). SM plays important roles in maintaining cell barrier functions and fluidity as a structural component of the cell membrane and regulate various cellular processes (Hannun \& Obeid, 2008; Ogretmen, 2018). Additionally, depending on the tumor biology, the sphingolipid level could be increased or decreased (Knapp, Chomicz, Swiderska, Chabowski, \& Jach, 2019). Thus, the decrease in SM levels could express the therapeutic effects of PP242. However, the association of DG in this study is not clearly understood. A summary of the main altered metabolic and lipidomic pathways due to three weeks of PP242 treatment in LS174T-induced colon cancer xenografts is shown in Fig. 8.

Herein, we investigated the antitumor effects of PP242 in LS174T cell-induced colon cancer xenograft mouse model's plasma and tumors using comprehensive metabolomics and lipidomics approaches to discover the metabolic mechanism of PP242 for the first time. Xenograft mice were treated with PP242 for three weeks, and significant reductions in tumor size and weight were observed. The plasma metabolic alterations were identical in the XC and PP242-treated groups, and no metabolites were significantly altered, suggesting that plasma samples were not enough to disclose the metabolic mechanism of PP242. The metabolic and lipidomic investigation of tumor tissues revealed that PP242 displayed its antitumor activity by inhibiting energy and glycerophospholipid metabolism, which are the major upregulated pathways in most cancers. PP242 also exerts its anticancer effect through inhibiting the $\beta$-oxidation of fatty acids. In addition, daily doses of PP242 over three weeks did not induce any abnormal effects, indicating its safety level. Together, this study provides valuable insights towards understanding the underlying actions of PP242 on the basis of the metabolome and could help to further implement PP242 in clinical analysis.

\section{Acknowledgements}


This study was supported by the Creative Fusion Research Program through the Creative Allied Project funded by the Korea Research Council of Fundamental Science and Technology (CAP-12-1-KIST), by the BioSynergy Research Project (NRF2013M3A9C4078145) of the Ministry of Science, ICT, and Future Planning through the National Research Foundation and by the KIST (Korea Institute of Science and Technology) Institutional Program (Project no. 2E30480).

\section{Author Contributions}

M.M.R conceived, designed and performed the experiments, analyzed and interpreted the data, and wrote the manuscript. H.L evaluated and checked the manuscript. B.H.J planned and managed all experiments, interpreted the results, and edited the manuscript.

\section{Conflict of Interest}

The authors declare no conflicts of interest.

\section{References}

Alfarouk, K. O., Verduzco, D., Rauch, C., Muddathir, A. K., Bashir, A. H. H., Elhassan, G. O. et al . (2014). Glycolysis, tumor metabolism, cancer growth and dissemination. A new pH-based etiopathogenic perspective and therapeutic approach to an old cancer question. Oncoscience, 1(22), 777-802.

Armitage, E. G., \& Southam, A. D. (2016). Monitoring cancer prognosis, diagnosis and treatment efficacy using metabolomics and lipidomics. Metabolomics, 12 , 146. doi:10.1007/s11306-016-1093-7.

Baba, Y., Tsukuda, M., Mochimatsu, I., Furukawa, S., Kagata, H., Nagashima, Y. et al . (2001). Cytostatic effect of inostamycin, an inhibitor of cytidine 5'-diphosphate 1,2-diacyl-sn-glycerol (CDP-DG): inositol transferase, on oral squamous cell carcinoma cell lines. Cell Biol Int, 25 (7), 613-620. doi:10.1006/cbir.2000.0706.

Baci, D., Bruno, A., Bassani, B., Tramacere, M., Mortara, L., Albini, A., \& Noonan, D. M. (2018). Acetyll-carnitine is an anti-angiogenic agent targeting the VEGFR2 and CXCR4 pathways. Cancer Lett, 429, 100-116. doi:10.1016/j.canlet.2018.04.018.

Blaser, B., Waselle, L., Dormond-Meuwly, A., Dufour, M., Roulin, D., Demartines, N., \& Dormond, O. (2012). Antitumor activities of ATP-competitive inhibitors of mTOR in colon cancer cells. BMC Cancer, 12, 86. doi:10.1186/1471-2407-12-86.

Bray, F., Ferlay, J., Soerjomataram, I., Siegel, R. L., Torre, L. A., \& Jemal, A. (2018). Global cancer statistics 2018: GLOBOCAN estimates of incidence and mortality worldwide for 36 cancers in 185 countries. CA Cancer J Clin, 68 (6), 394-424. doi:10.3322/caac.21492.

Cala, M. P., Aldana, J., Medina, J., Sanchez, J., Guio, J., Wist, J., \& Meesters, R. J. W. (2018). Multiplatform plasma metabolic and lipid fingerprinting of breast cancer: A pilot control-case study in Colombian Hispanic women. PLoS One, 13 (2), e0190958. doi:10.1371/journal.pone.0190958.

Cheng, L., Xia, Z., Bian, X., Li, G., Hu, J., Cao, Y. et al . (2015). Combination of cetuximab and PP242 synergistically suppress the progression of wild-type KRAS colorectal carcinoma. Onco Targets Ther, 8 , 3185-3192. doi:10.2147/OTT.S82453.

Cheng, M., Bhujwalla, Z. M., \& Glunde, K. (2016). Targeting Phospholipid Metabolism in Cancer. Front Oncol, 6 , 266. doi:10.3389/fonc.2016.00266.

Davis, H. W., Vallabhapurapu, S. D., Chu, Z., Vallabhapurapu, S. L., Franco, R. S., Mierzwa, M. et al . (2019). Enhanced phosphatidylserine-selective cancer therapy with irradiation and SapC-DOPS nanovesicles. Oncotarget, 10(8), 856-868.

de la Cruz-Lopez, K. G., Castro-Munoz, L. J., Reyes-Hernandez, D. O., Garcia-Carranca, A., \& ManzoMerino, J. (2019). Lactate in the Regulation of Tumor Microenvironment and Therapeutic Approaches. Front Oncol, 9 , 1143. doi:10.3389/fonc.2019.01143. 
Duan, R.-D. (2016). Phospholipid signals and intestinal carcinogenesis. Scandinavian Journal of Food and Nutrition, 50 (sup2), 45-53. doi:10.1080/17482970601075703.

Falkenburger, B. H., Jensen, J. B., Dickson, E. J., Suh, B. C., \& Hille, B. (2010). Phosphoinositides: lipid regulators of membrane proteins. J Physiol, 588 (Pt 17), 3179-3185. doi:10.1113/jphysiol.2010.192153.

Francipane, M. G., \& Lagasse, E. (2013). mTOR pathway in colorectal cancer: an update. Oncotarget, $5(1), 49-66$.

Furse, S., \& de Kroon, A. I. (2015). Phosphatidylcholine's functions beyond that of a membrane brick. Mol Membr Biol, 32 (4), 117-119. doi:10.3109/09687688.2015.1066894.

Gao, D., Wang, Y., Xie, W., Yang, T., Jiang, Y., Guo, Y. et al . (2016). Metabolomics study on the antitumor effect of marine natural compound flexibilide in HCT-116 colon cancer cell line. J Chromatogr B, 1014 , 17-23. doi:10.1016/j.jchromb.2016.01.003.

Gibellini, F., \& Smith, T. K. (2010). The Kennedy pathway-De novo synthesis of phosphatidylethanolamine and phosphatidylcholine. IUBMB Life, 62 (6), 414-428. doi:10.1002/iub.337.

Goetze, K., Walenta, S., Ksiazkiewicz, M., Kunz-Schughart, L. A., \& Mueller-Klieser, W. (2011). Lactate enhances motility of tumor cells and inhibits monocyte migration and cytokine release. Int J Oncol, 39 (2), 453-463. doi:10.3892/ijo.2011.1055.

Gulhati, P., Cai, Q., Li, J., Liu, J., Rychahou, P. G., Qiu, S. et al . (2009). Targeted inhibition of mammalian target of rapamycin signaling inhibits tumorigenesis of colorectal cancer. Clin Cancer Res, 15 (23), 7207-7216. doi:10.1158/1078-0432.CCR-09-1249.

Hannun, Y. A., \& Obeid, L. M. (2008). Principles of bioactive lipid signalling: lessons from sphingolipids. Nat Rev Mol Cell Biol, 9 (2), 139-150. doi:10.1038/nrm2329.

Hay, N. (2016). Reprogramming glucose metabolism in cancer: can it be exploited for cancer therapy? Nat Rev Cancer, 16 (10), 635-649. doi:10.1038/nrc.2016.77.

Imoto, M., Tanabe, K., Simizu, S., Tashiro, E., Takada, M., \& Umezawa, K. (1998). Inhibition of Cyclin D1 Expression and Induction of Apoptosis by Inostamycin in Small Cell Lung Carcinoma Cells. Jpn J Cancer Res, 89, 315-322.

Janes, M. R., Limon, J. J., So, L., Chen, J., Lim, R. J., Chavez, M. A.et al . (2010). Effective and selective targeting of leukemia cells using a TORC1/2 kinase inhibitor. Nat Med, 16 (2), 205-213. doi:10.1038/nm.2091.

Jin, L., Alesi, G. N., \& Kang, S. (2016). Glutaminolysis as a target for cancer therapy. Oncogene, 35 (28), 3619-3625. doi:10.1038/onc.2015.447.

Johnson, S. C., Rabinovitch, P. S., \& Kaeberlein, M. (2013). mTOR is a key modulator of ageing and age-related disease. Nature, 493 (7432), 338-345. doi:10.1038/nature11861.

Jones, D. T., Valli, A., Haider, S., Zhang, Q., Smethurst, E. A., Schug, Z. T. et al . (2019). 3D Growth of Cancer Cells Elicits Sensitivity to Kinase Inhibitors but Not Lipid Metabolism Modifiers. Mol Cancer Ther, 18 (2), 376-388. doi:10.1158/1535-7163.MCT-17-0857.

Kim, H. Y., Jin, H., Bae, J., \& Choi, H. K. (2019). Metabolic and lipidomic investigation of the antiproliferative effects of coronatine against human melanoma cells. Sci Rep, 9 (1), 3140. doi:10.1038/s41598-01939990-w.

Knapp, P., Chomicz, K., Swiderska, M., Chabowski, A., \& Jach, R. (2019). Unique Roles of Sphingolipids in Selected Malignant and Nonmalignant Lesions of Female Reproductive System. Biomed Res Int, 2019 , 4376583. doi:10.1155/2019/4376583. 
Kurabe, N., Hayasaka, T., Ogawa, M., Masaki, N., Ide, Y., Waki, M.et al . (2013). Accumulated phosphatidylcholine (16:0/16:1) in human colorectal cancer; possible involvement of LPCAT4. Cancer Sci, 104 (10), 1295-1302. doi:10.1111/cas.12221.

Laplante, M., \& Sabatini, D. M. (2012). mTOR signaling in growth control and disease. Cell, 149 (2), 274-293. doi:10.1016/j.cell.2012.03.017.

Law, S. H., Chan, M. L., Marathe, G. K., Parveen, F., Chen, C. H., \& Ke, L. Y. (2019). An Updated Review of Lysophosphatidylcholine Metabolism in Human Diseases. Int J Mol Sci, 20 (5). doi:10.3390/ijms20051149.

Lu, S., Lu, R., Song, H., Wu, J., Liu, X., Zhou, X. et al . (2019). Metabolomic study of natrin-induced apoptosis in SMMC-7721 hepatocellular carcinoma cells by ultra-performance liquid chromatography-quadrupole/time-of-flight mass spectrometry. Int J Biol Macromol, 124 , 1264-1273. doi:10.1016/j.ijbiomac.2018.11.060.

Mason, E. F., \& Rathmell, J. C. (2011). Cell metabolism: an essential link between cell growth and apoptosis. Biochim Biophys Acta, 1813 (4), 645-654. doi:10.1016/j.bbamcr.2010.08.011.

Mecca, C., Giambanco, I., Bruscoli, S., Bereshchenko, O., Fioretti, B., Riccardi, C. et al . (2018). PP242 Counteracts Glioblastoma Cell Proliferation, Migration, Invasiveness and Stemness Properties by Inhibiting mTORC2/AKT. Front Cell Neurosci, 12 , 99. doi:10.3389/fncel.2018.00099.

Melone, M. A. B., Valentino, A., Margarucci, S., Galderisi, U., Giordano, A., \& Peluso, G. (2018). The carnitine system and cancer metabolic plasticity. Cell Death Dis, 9 (2), 228. doi:10.1038/s41419-018-03137 .

Murfitt, S. A., Zaccone, P., Wang, X., Acharjee, A., Sawyer, Y., Koulman, A. et al . (2018). Metabolomics and Lipidomics Study of Mouse Models of Type 1 Diabetes Highlights Divergent Metabolism in Purine and Tryptophan Metabolism Prior to Disease Onset. J Proteome Res, 17 (3), 946-960. doi:10.1021/acs.jproteome.7b00489.

Ogretmen, B. (2018). Sphingolipid metabolism in cancer signalling and therapy. Nat Rev Cancer, 18 (1), 33-50. doi:10.1038/nrc.2017.96.

Patel, D., \& Witt, S. N. (2017). Ethanolamine and Phosphatidylethanolamine: Partners in Health and Disease. Oxid Med Cell Longev, 2017 , 4829180. doi:10.1155/2017/4829180.

Qiu, Y., Cai, G., Su, M., Chen, T., Zheng, X., Xu, Y. et al . (2009). Serum Metabolite Profiling of Human Colorectal Cancer Using GC-TOFMS and UPLC-QTOFMS. J Proteome Res, 8, 4844-4850.

Qu, Q., Zeng, F., Liu, X., Wang, Q. J., \& Deng, F. (2016). Fatty acid oxidation and carnitine palmitoyltransferase I: emerging therapeutic targets in cancer. Cell Death Dis, 7 , e2226. doi:10.1038/cddis.2016.132.

Rivera-Velez, S. M., Broughton-Neiswanger, L. E., Suarez, M. A., Slovak, J. E., Hwang, J. K., Navas, J. et al . (2019). Understanding the effect of repeated administration of meloxicam on feline renal cortex and medulla: A lipidomics and metabolomics approach. J Vet Pharmacol Ther, 42 (4), 476-486. doi:10.1111/jvp.12788.

Roulin, D., Cerantola, Y., Dormond-Meuwly, A., Demartines, N., \& Dormond, O. (2010). Targeting mTORC2 inhibits colon cancer cell proliferation in vitro and tumor formation in vivo. Mol Cancer, 9 , 57. doi:10.1186/1476-4598-9-57.

Sarbassov, D. D., Ali, S. M., Sengupta, S., Sheen, J. H., Hsu, P. P., Bagley, A. F. et al . (2006). Prolonged rapamycin treatment inhibits mTORC2 assembly and Akt/PKB. Mol Cell, 22 (2), 159-168. doi:10.1016/j.molcel.2006.03.029.

Stafford, J. H., \& Thorpe, P. E. (2011). Increased exposure of phosphatidylethanolamine on the surface of tumor vascular endothelium. Neoplasia, 13 (4), 299-308. doi:10.1593/neo.101366. 
Takahashi, M., Okazaki, H., Ogata, Y., Takeuchi, K., Ikeda, U., Shimada, K. (2001). Lysophosphatidylcholine induces apoptosis in human endothelial cells through a p38-mitogen-activated protein kinasedependent mechanism. Atherosclerosis, 161(2002), 387-384.

Tan, L. T., Chan, K. G., Pusparajah, P., Lee, W. L., Chuah, L. H., Khan, T. M. et al . (2017). Targeting Membrane Lipid a Potential Cancer Cure? Front Pharmacol, 8 , 12. doi:10.3389/fphar.2017.00012.

Tanaka, K., Sasayama, T., Irino, Y., Takata, K., Nagashima, H., Satoh, N. et al . (2015). Compensatory glutamine metabolism promotes glioblastoma resistance to mTOR inhibitor treatment. J Clin Invest, 125 (4), 1591-1602. doi:10.1172/JCI78239.

Tian, T., Li, X., \& Zhang, J. (2019). mTOR Signaling in Cancer and mTOR Inhibitors in Solid Tumor Targeting Therapy. Int J Mol Sci, 20 (3). doi:10.3390/ijms20030755.

Tsang, C. K., Qi, H., Liu, L. F., \& Zheng, X. F. (2007). Targeting mammalian target of rapamycin (mTOR) for health and diseases. Drug Discov Today, 12 (3-4), 112-124. doi:10.1016/j.drudis.2006.12.008.

Vallabhapurapu, S.D., Blanco, V. M., Sulaiman, M. K., Vallabhapurapu, S. L., Chu, Z., Franco RS et al - (2015). Variation in human cancer cell external phosphatidylserine is regulated by flippase activity and intracellular calcium. Oncotarget, 6(33), 34375-34388.

Wang, X. W., \& Zhang, Y. J. (2014). Targeting mTOR network in colorectal cancer therapy. World J Gastroenterol, 20 (15), 4178-4188. doi:10.3748/wjg.v20.i15.4178.

Warburg, B. (1956). On the Origin of Cancer Cells. Science, 123(3191), 309-314. DOI: 10.1126/science.123.3191.309.

Xing, X., Zhang, L., Wen, X., Wang, X., Cheng, X., Du, H. et al . (2014). PP242 suppresses cell proliferation, metastasis, and angiogenesis of gastric cancer through inhibition of the PI3K/AKT/mTOR pathway. Anticancer Drugs, 25 (10), 1129-1140. doi:10.1097/CAD.0000000000000148.

Zeng, Z., Shi, Y. X., Tsao, T., Qiu, Y., Kornblau, S. M., Baggerly, K. A. et al . (2012). Targeting of mTORC1/2 by the mTOR kinase inhibitor PP242 induces apoptosis in AML cells under conditions mimicking the bone marrow microenvironment. Blood, 120 (13), 2679-2689. doi:10.1182/blood-2011-11-393934.

Zhang, Y. J., Dai, Q., Sun, D. F., Xiong, H., Tian, X. Q., Gao, F. H.et al . (2009). mTOR signaling pathway is a target for the treatment of colorectal cancer. Ann Surg Oncol, 16 (9), 2617-2628. doi:10.1245/s10434009-0555-9.

Zhang, Z., Zhang, G., Kong, C., \& Gong, D. (2016). PP242 suppresses bladder cancer cell proliferation and migration through deactivating the mammalian target of rapamycin complex 2/AKT1 signaling pathway. Mol Med Rep, 13 (1), 333-338. doi:10.3892/mmr.2015.4528.

Zheng, Z., Xu, L., Zhang, S., Li, W., Tou, F., He, Q. et al . (2017). Peiminine inhibits colorectal cancer cell proliferation by inducing apoptosis and autophagy and modulating key metabolic pathways. Oncotarget, 8 (29), 47619-47631. doi:10.18632/oncotarget.17411.

Table 1. List of altered identified metabolites in plasma due to the effects of PP242 using metabolomics and lipidomics approaches.

\begin{tabular}{llllllll}
\hline No. & Class & Metabolite Name & Exact mass & $\mathrm{m} / \mathrm{z}$ & $\mathrm{RT}$ & $\begin{array}{l}\text { XC vs NC } \\
\text { Trend }\end{array}$ & $\begin{array}{l}\text { XC vs NC } \\
\text { Fold change }\end{array}$ \\
1 & Carboxylic acids and derivatives & Creatine & 131.07 & 132.08 & 0.88 & -* & 0.57 \\
2 & Hydroxy acids and derivatives & L-Lactic acid & 90.03 & 89.02 & 0.93 & -* & 3.32 \\
3 & Indoles and derivatives & Indoleacrylic acid & 187.06 & 188.07 & 4.83 & -* & 0.56 \\
4 & Fatty acyls & L-Palmitoylcarnitine & 399.33 & 400.34 & 12.41 & -** & 0.36 \\
5 & Fatty acyls & Vaccenyl carnitine & 425.35 & 426.36 & 12.59 & -* & 0.21 \\
6 & Fatty acyls & Eicosapentaenoic acid & 302.22 & 301.22 & 3.42 & -* & 5.58
\end{tabular}




$\begin{array}{ll}7 & \text { Glycerophospholipids } \\ 8 & \text { Glycerophospholipids } \\ 9 & \text { Glycerophospholipids } \\ 10 & \text { Glycerophospholipids } \\ 11 & \text { Glycerophospholipids } \\ 12 & \text { Glycerophospholipids } \\ 13 & \text { Glycerophospholipids } \\ 14 & \text { Glycerophospholipids } \\ 15 & \text { Glycerophospholipids } \\ 16 & \text { Glycerophospholipids } \\ 17 & \text { Glycerophospholipids } \\ 18 & \text { Glycerophospholipids } \\ 19 & \text { Glycerophospholipids } \\ 20 & \text { Glycerophospholipids } \\ 21 & \text { Glycerophospholipids } \\ 22 & \text { Glycerophospholipids }\end{array}$

LysoPC(16:0)
LysoPC(18:0)
LysoPC $(20: 3)$
LysoPC $(22: 6)$
PC $(35: 4)$
PC $(36: 4)$
PC $(36: 1)$
PC $(37: 4)$
PC $(38: 5)$
PC $(38: 4)$
PC $(38: 3)$
PC $(38: 2)$
LyosPE $(20: 4)$
LysoPE $(22: 6)$
PE( $(38: 4)$
PG $(42: 2)$

495.33
523.36
545.34
567.33
767.54
781.56
787.61
795.58
807.57
809.59
811.61
813.63
501.28
525.28
767.54
858.52

$\begin{array}{llll}518.32 & 3.82 & \text {-** } & 3.05 \\ 524.37 & 5.37 & \text { ** }^{*} & 1.29 \\ 546.35 & 13.67 & \text { - } & 2.48 \\ 590.32 & 13.28 & \text {-*** } & 1.92 \\ 768.55 & 9.21 & \text {-*** } & 0.57 \\ 782.57 & 8.54 & \text {-** } & 0.55 \\ 788.62 & 9.49 & \text { - } & 1.29 \\ 796.58 & 8.81 & \text {-* } & 0.58 \\ 808.58 & 8.60 & \text { - } & 1.22 \\ 810.60 & 17.17 & \text { - } & 0.79 \\ 812.62 & 9.28 & \text {-* } & 1.54 \\ 814.63 & 9.60 & \text { - } & 1.11 \\ 500.27 & 13.34 & \text {-** } & 1.52 \\ 524.27 & 13.31 & \text {-* } & 1.51 \\ 766.54 & 9.22 & \text { - } & 0.66 \\ 857.51 & 7.91 & - & 1.44\end{array}$

${ }^{*} \mathrm{P}<0.05 ;{ }^{* *} \mathrm{P}<0.01 ; * * * \mathrm{P}<0.001$

Table 2. List of altered identified metabolites in plasma due to the effects of PP242 using metabolomics and lipidomics approaches.

\begin{tabular}{|c|c|c|c|c|c|c|}
\hline No. & Class & Metabolite Name & Exact mass & $\mathrm{m} / \mathrm{z}$ & $\mathrm{RT}$ & $\begin{array}{l}\text { XC vs PP242 } \\
\text { Trend }\end{array}$ \\
\hline 1 & Amino acid & L-Methionine & 149.05 & 150.06 & 1.21 & —* \\
\hline 2 & Carboxylic acids and derivatives & Creatine & 131.07 & 132.08 & 0.91 & —* \\
\hline 3 & Carboxylic acids and derivatives & L-Aspartic acid & 133.04 & 132.03 & 1.66 & —* \\
\hline 4 & Hydroxy acids and derivatives & L-Lactic acid & 90.03 & 89.02 & 0.88 & —* \\
\hline 5 & Purine nucleosides & Inosine & 268.08 & 267.07 & 1.50 & —** \\
\hline 6 & Pyrimidine nucleotides & Uridine 5'-monophosphate & 324.03 & 323.03 & 1.17 & —* \\
\hline 7 & Phenylpropanoic acids & Phenyllactic acid & 166.06 & 165.05 & 7.12 & —* \\
\hline 8 & Fatty Acyls & L-Carnitine & 161.11 & 162.11 & 0.88 & —*** \\
\hline 9 & Fatty Acyls & L-Acetylcarnitine & 203.12 & 204.12 & 1.11 & —* \\
\hline 10 & Fatty Acyls & L-Palmitoylcarnitine & 399.34 & 400.34 & 12.31 & —* \\
\hline 11 & Fatty Acyls & Vaccenic acid & 282.26 & 281.25 & 5.41 & —* \\
\hline 12 & Fatty Acyls & Arachidonic acid & 304.24 & 303.24 & 4.17 & —** \\
\hline 13 & Fatty Acyls & 5-HEPE & 318.22 & 317.21 & 12.11 & —** \\
\hline 14 & Fatty Acyls & 7-HETE & 320.23 & 319.23 & 12.58 & —** \\
\hline 15 & Fatty Acyls & 15(S)-Hydroxyeicosatrienoic acid & 322.25 & 321.24 & 12.81 & —** \\
\hline 16 & Fatty Acyls & $9,12,13$-TriHOME & 330.24 & 329.23 & 10.07 & —*** \\
\hline 17 & Fatty Acyls & 16-HDoHE & 344.23 & 343.23 & 12.56 & $\ldots * *$ \\
\hline 18 & Glycerophospholipids & LysoPC (18:2) & 519.33 & 520.34 & 13.31 & —* \\
\hline 19 & Glycerophospholipids & $\mathrm{PC}(30: 0)$ & 705.53 & 706.54 & 8.26 & —* \\
\hline 20 & Glycerophospholipids & $\mathrm{PC}(32: 2)$ & 729.53 & 730.54 & 7.92 & — \\
\hline 21 & Glycerophospholipids & $\mathrm{PC}(32: 1)$ & 731.55 & 732.55 & 8.38 & —* \\
\hline 22 & Glycerophospholipids & $\mathrm{PC}(32: 0)$ & 733.56 & 734.57 & 8.81 & —* \\
\hline 23 & Glycerophospholipids & $\mathrm{PC}(33: 1)$ & 745.56 & 746.57 & 8.67 & —* \\
\hline 24 & Glycerophospholipids & $\mathrm{PC}(34: 3)$ & 755.55 & 756.55 & 8.21 & —* \\
\hline 25 & Glycerophospholipids & $\mathrm{PC}(34: 2)$ & 757.56 & 758.57 & 8.49 & —* \\
\hline 26 & Glycerophospholipids & $\mathrm{PC}(34: 1)$ & 759.58 & 760.58 & 8.89 & —** \\
\hline 27 & Glycerophospholipids & $\mathrm{PC}(36: 5)$ & 779.55 & 780.56 & 8.07 & —* \\
\hline
\end{tabular}




\begin{tabular}{ll}
28 & Glycerophospholipids \\
29 & Glycerophospholipids \\
30 & Glycerophospholipids \\
31 & Glycerophospholipids \\
32 & Glycerophospholipids \\
33 & Glycerophospholipids \\
34 & Glycerophospholipids \\
35 & Glycerophospholipids \\
36 & Glycerophospholipids \\
37 & Glycerophospholipids \\
38 & Glycerophospholipids \\
39 & Glycerophospholipids \\
40 & Glycerophospholipids \\
41 & Glycerophospholipids \\
42 & Glycerophospholipids \\
43 & Glycerophospholipids \\
44 & Glycerophospholipids \\
45 & Glycerophospholipids \\
46 & Glycerophospholipids \\
47 & Glycerophospholipids \\
48 & Glycerophospholipids \\
49 & Glycerophospholipids \\
50 & Glycerophospholipids \\
51 & Glycerophospholipids \\
52 & Glycerophospholipids \\
53 & Glycerophospholipids \\
54 & Glycerophospholipids \\
55 & Glycerophospholipids \\
56 & Glycerophospholipids \\
57 & Glycerophospholipids \\
58 & Glycerolipids \\
59 & Sphingolipids \\
\hline &
\end{tabular}

\begin{tabular}{|c|}
\hline$(36: 4)$ \\
\hline $\mathrm{PC}(36: 3)$ \\
\hline $\mathrm{PC}(36: 2)$ \\
\hline $\mathrm{PC}(38: 4)$ \\
\hline $\mathrm{PE}(34: 2)$ \\
\hline $\mathrm{PE}(34: 1)$ \\
\hline $\operatorname{PE}(36: 4)$ \\
\hline $\operatorname{PE}(36: 3)$ \\
\hline $\operatorname{PE}(36: 1)$ \\
\hline $\operatorname{PE}(38: 6)$ \\
\hline $\mathrm{PE}(38: 5)$ \\
\hline $\mathrm{PE}(38: 4)$ \\
\hline $\mathrm{PE}(\mathrm{P}-34: 2)$ \\
\hline $\mathrm{PE}(\mathrm{P}-34: 1)$ \\
\hline $\mathrm{PE}(\mathrm{P}-36: 4)$ \\
\hline $\mathrm{PE}(\mathrm{P}-36: 2)$ \\
\hline $\mathrm{PE}(\mathrm{P}-36: 0)$ \\
\hline $\mathrm{PE}(\mathrm{P}-38: 6)$ \\
\hline $\mathrm{PE}(\mathrm{P}-38: 4)$ \\
\hline $\mathrm{PE}(\mathrm{P}-38: 3)$ \\
\hline $\mathrm{PE}(\mathrm{P}-40: 6)$ \\
\hline $\mathrm{PI}(34: 2)$ \\
\hline $\mathrm{PI}(34: 1)$ \\
\hline PI(36:4) \\
\hline $\operatorname{PI}(36: 3)$ \\
\hline PI(38:3) \\
\hline PS(36:2) \\
\hline PS(36:1) \\
\hline $\operatorname{PS}(40: 1)$ \\
\hline $\operatorname{PS}(42: 1)$ \\
\hline DG(36:2) \\
\hline $\operatorname{sM}(34: 1$ \\
\hline
\end{tabular}

\begin{tabular}{|c|c|c|c|}
\hline 781.56 & 782.57 & 8.42 & —* \\
\hline 783.58 & 784.58 & 8.66 & —** \\
\hline 785.59 & 786.60 & 9.03 & —* \\
\hline 809.59 & 810.60 & 8.70 & —** \\
\hline 715.51 & 714.51 & 8.65 & —* \\
\hline 717.53 & 716.52 & 9.03 & —* \\
\hline 739.51 & 738.51 & 8.58 & —* \\
\hline 741.53 & 742.54 & 8.71 & —** \\
\hline 745.56 & 744.55 & 9.52 & —* \\
\hline 763.51 & 762.51 & 8.46 & —** \\
\hline 765.53 & 764.52 & 8.65 & —** \\
\hline 767.55 & 766.54 & 9.10 & —* \\
\hline 699.52 & 698.51 & 8.94 & —* \\
\hline 701.53 & 702.54 & 9.32 & — $* * *$ \\
\hline 723.52 & 724.53 & 8.87 & —** \\
\hline 727.55 & 726.54 & 9.46 & —* \\
\hline 731.58 & 730.57 & 9.85 & —* \\
\hline 747.52 & 746.51 & 8.73 & —** \\
\hline 751.55 & 752.56 & 9.38 & —* \\
\hline 753.57 & 754.57 & 9.50 & —* \\
\hline 775.55 & 776.56 & 9.26 & —* \\
\hline 834.52 & 833.52 & 7.83 & —* \\
\hline 836.54 & 835.53 & 8.21 & —** \\
\hline 858.53 & 857.52 & 7.77 & —* \\
\hline 860.54 & 859.53 & 7.99 & —** \\
\hline 888.57 & 887.56 & 8.51 & — $* * *$ \\
\hline 787.54 & 786.53 & 8.42 & —* \\
\hline 789.55 & 790.56 & 8.80 & —** \\
\hline 845.61 & 844.61 & 9.72 & —* \\
\hline 873.64 & 872.64 & 10.12 & —* \\
\hline 620.54 & 621.55 & 17.97 & — $* * *$ \\
\hline 702.57 & 703.57 & 8.25 & —* \\
\hline
\end{tabular}

${ }^{*} \mathrm{P}<0.05 ;{ }^{* *} \mathrm{P}<0.01 ;{ }^{* * *} \mathrm{P}<0.00$

\section{Hosted file}

image1.emf available at https://authorea.com/users/307261/articles/438218-evaluation-of-theantitumor-effects-of-pp242-in-an-ls174t-cell-induced-colon-cancer-xenograft-model-usingcomprehensive-metabolomics-and-lipidomics

Fig. 1. Body weight changes of normal control (NC; non tumor-bearing), xenograft control (XC; treated with vehicle), and PP242 treatment (treated with PP242 at a dose of $60 \mathrm{mg} \cdot \mathrm{kg}^{-1}$.day ${ }^{-1}$ ) group mice for three weeks. ${ }^{*} \mathrm{p}<0.01,{ }^{* *} \mathrm{p}<0.01[\mathrm{NC}$ vs PP242 treated].

\section{Hosted file}

image2.emf available at https://authorea.com/users/307261/articles/438218-evaluation-of-theantitumor-effects-of-pp242-in-an-ls174t-cell-induced-colon-cancer-xenograft-model-usingcomprehensive-metabolomics-and-lipidomics

Fig. 2. PP242 treatment showed tumor growth inhibition in vivo. (A) Tumor growth (in the right flank region) rate of LS174T cells induced colon cancer xenograft model after treating with vehicle (xenograft control) and PP242 (PP242 treated; $60 \mathrm{mg} \cdot \mathrm{kg}^{-1}$. day ${ }^{-1}$ ) for three weeks. Treatment was started when the 
tumor sized reached $150 \sim 200 \mathrm{~mm}^{3}$. Tumor volume was presented as mean \pm SD. (B) Representative images of tumor weight of xenograft control (vehicle) and PP242 treated. (C) Mean weight of harvested tumor after the three weeks treatment of PP242. ${ }^{*} \mathrm{p}<0.05 ;{ }^{* *} \mathrm{p}<0.01 ;{ }^{* * *} \mathrm{p}<0.001 ;(\mathrm{n}=5$ per group).

\section{Hosted file}

image3.emf available at https://authorea.com/users/307261/articles/438218-evaluation-of-theantitumor-effects-of-pp242-in-an-ls174t-cell-induced-colon-cancer-xenograft-model-usingcomprehensive-metabolomics-and-lipidomics

Fig. 3. Evaluated serum biochemical parameters of normal control (NC), xenograft control (XC; vehicle treated), and PP242 treated to check the adverse effects associated with PP242. Error bar was presented as mean \pm SD. ${ }^{*} \mathrm{p}<0.05 ;{ }^{*} \mathrm{p}<0.01 ;{ }^{* * *} \mathrm{p}<0.001 ;(\mathrm{n}=5$ per group $)$.

\section{Hosted file}

image4.emf available at https://authorea.com/users/307261/articles/438218-evaluation-of-theantitumor-effects-of-pp242-in-an-ls174t-cell-induced-colon-cancer-xenograft-model-usingcomprehensive-metabolomics-and-lipidomics

Fig. 4. PLS-DA score plot of plasma samples after three weeks treatment of PP242 at a dose of 60 mg.kg- ${ }^{-1}$.day ${ }^{-1}$. (A) Metabolomics $\left(\mathrm{ES}+; \mathrm{R}^{2}=0.43, \mathrm{Q}^{2}=0.1\right.$ ), (B) metabolomics (ES-; $\mathrm{R}^{2}=0.43, \mathrm{Q}^{2}$ $=0.21),(\mathrm{C})$ Lipidomics $\left(\mathrm{ES}+; \mathrm{R}^{2}=0.61, \mathrm{Q}^{2}=0.20\right)$, and Lipidomics $\left(\mathrm{ES}-; \mathrm{R}^{2}=0.51, \mathrm{Q}^{2}=0.17\right)$.

\section{Hosted file}

image5.emf available at https://authorea.com/users/307261/articles/438218-evaluation-of-theantitumor-effects-of-pp242-in-an-ls174t-cell-induced-colon-cancer-xenograft-model-usingcomprehensive-metabolomics-and-lipidomics

Fig. 5. PLS-DA score plot of tumor tissue samples after three weeks treatment of PP242 at a dose of 60 mg. $\mathrm{kg}^{-1}$.day ${ }^{-1}$. (A) Metabolomics $\left(\mathrm{ES}+; \mathrm{R}^{2}=0.89, \mathrm{Q}^{2}=0.60\right),(\mathrm{B})$ metabolomics $\left(\mathrm{ES}-; \mathrm{R}^{2}=0.94, \mathrm{Q}^{2}=\right.$ $0.52)$, (C) Lipidomics (ES+; $\left.\mathrm{R}^{2}=0.99, \mathrm{Q}^{2}=0.87\right)$, and Lipidomics $\left(\mathrm{ES}-; \mathrm{R}^{2}=0.82, \mathrm{Q}^{2}=0.04\right)$.

\section{Hosted file}

image6.emf available at https://authorea.com/users/307261/articles/438218-evaluation-of-theantitumor-effects-of-pp242-in-an-ls174t-cell-induced-colon-cancer-xenograft-model-usingcomprehensive-metabolomics-and-lipidomics

Fig. 6. Heat map of significantly altered metabolites in plasma of normal control (NC), xenograft control (XC, tumor bearing mice treated only with vehicle), and PP242 treated (treated with PP242 at a dose of 60 $\mathrm{mg} \cdot \mathrm{kg}^{-1} \cdot \mathrm{day}^{-1}$ ) groups after the three weeks experiment period.

\section{Hosted file}

image7.emf available at https://authorea.com/users/307261/articles/438218-evaluation-of-theantitumor-effects-of-pp242-in-an-ls174t-cell-induced-colon-cancer-xenograft-model-usingcomprehensive-metabolomics-and-lipidomics

Fig. 7. Heat map of significantly altered metabolites in tumor tissues of (XC, tumor bearing mice treated only with vehicle), and PP242 treated (treated with PP242 at a dose of $60 \mathrm{mg} \cdot \mathrm{kg}^{-1}$. day ${ }^{-1}$ ) groups after the three weeks experiment period.

\section{Hosted file}

image8.emf available at https://authorea.com/users/307261/articles/438218-evaluation-of-theantitumor-effects-of-pp242-in-an-ls174t-cell-induced-colon-cancer-xenograft-model-usingcomprehensive-metabolomics-and-lipidomics 
Fig. 8. Overview of affected pathways of identified 59 significantly altered metabolites after the treatment with second generation mTOR inhibitor (PP242) at an oral dose of $60 \mathrm{mg} \cdot \mathrm{kg}^{-1}$.day-1 for three weeks. The major perturbed metabolic pathways associated with PP242 effects were energy metabolism, fatty acid metabolism and glycerophospholipid metabolism. Grey color represents increased metabolites after the mTOR inhibition by PP242; dark grey color represents decreased metabolites after the mTOR inhibition by PP242 [grey and dark grey box indicates the identified metabolites that are significantly altered].

Figure. 1.

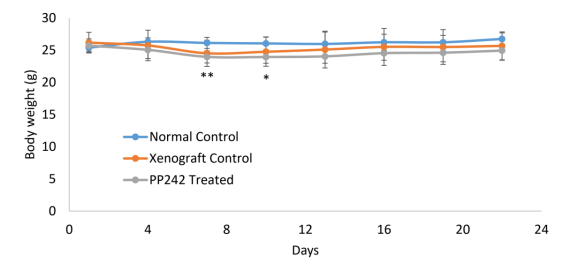

Figure. 2.

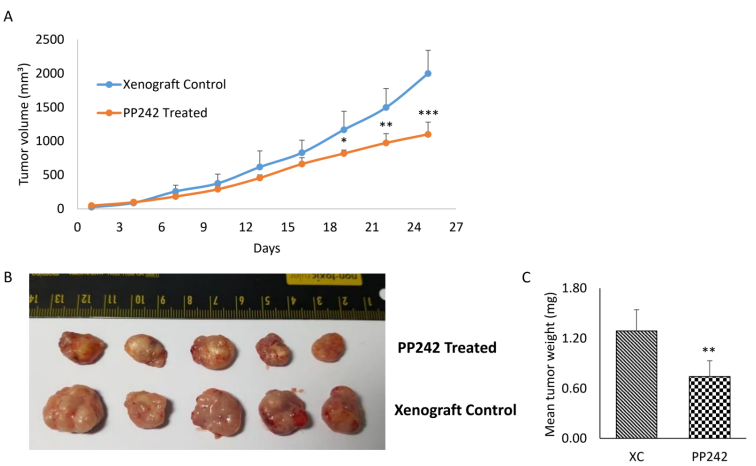


Figure. 3.

Liver toxicity marker
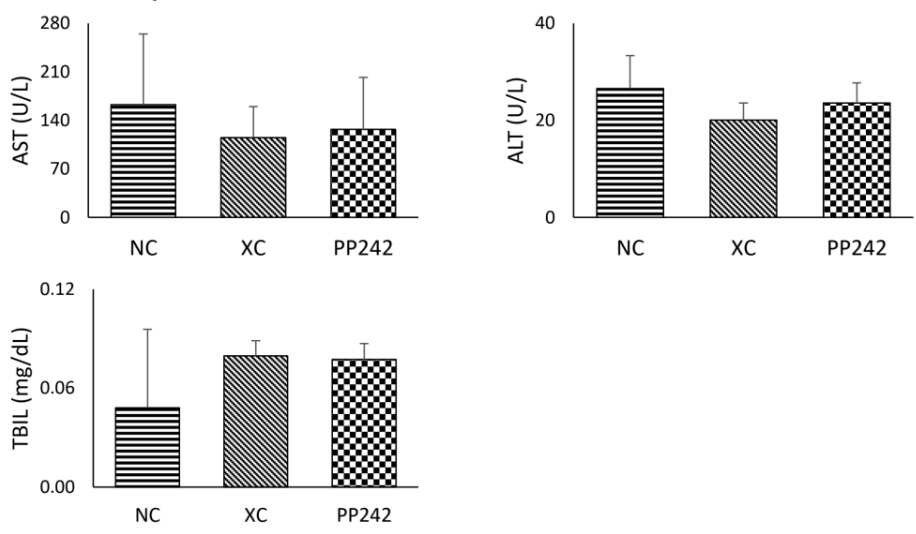

Kidney toxicity marker
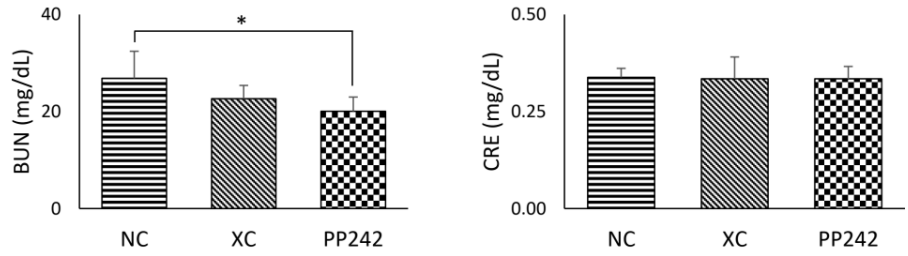

Heart toxicity marker
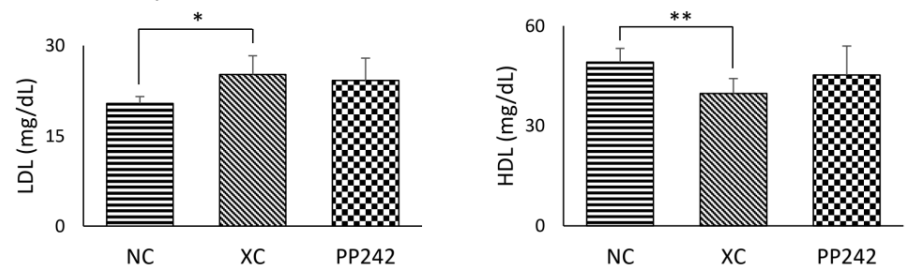

Figure. 4.

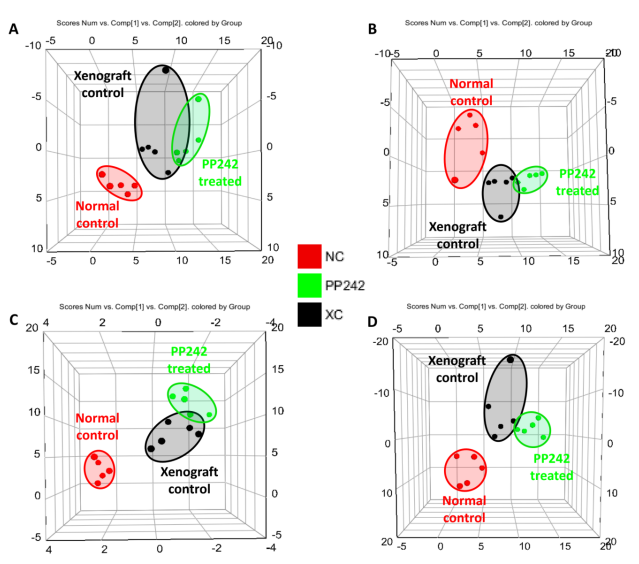


Figure. 5 .
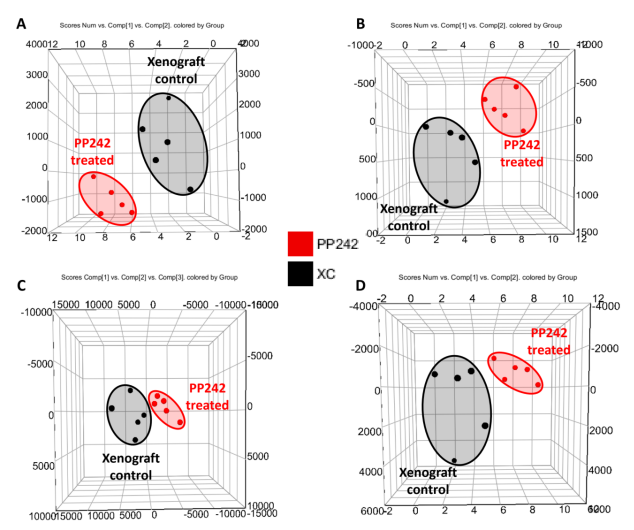

Figure. 6.

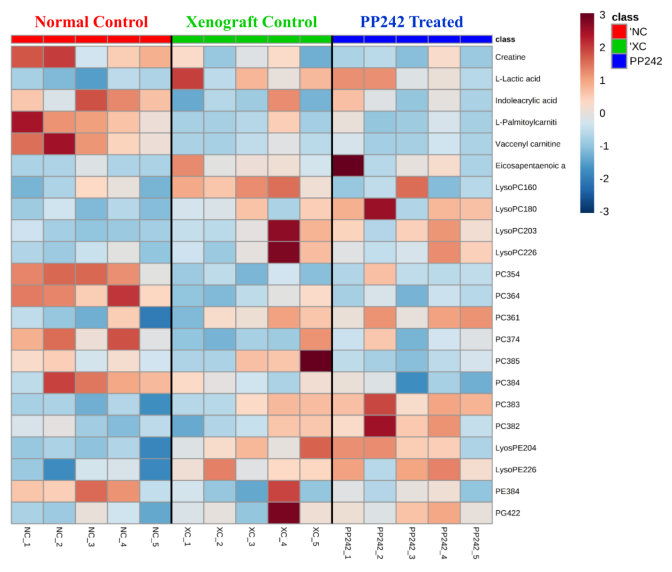

Figure. 7.

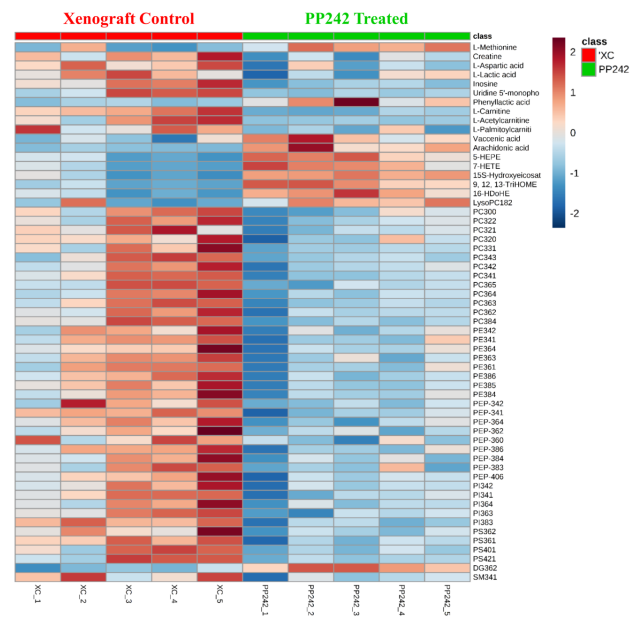




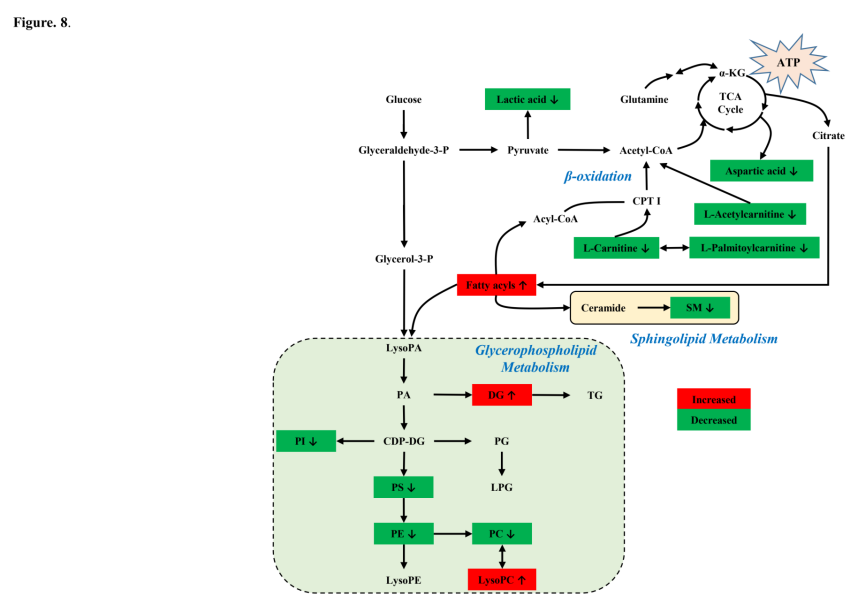

\section{Hosted file}

Table. 1.pptx available at https://authorea.com/users/307261/articles/438218-evaluation-ofthe-antitumor-effects-of-pp242-in-an-ls174t-cell-induced-colon-cancer-xenograft-modelusing-comprehensive-metabolomics-and-lipidomics

\section{Hosted file}

Table. 2.pptx available at https://authorea.com/users/307261/articles/438218-evaluation-ofthe-antitumor-effects-of-pp242-in-an-ls174t-cell-induced-colon-cancer-xenograft-modelusing-comprehensive-metabolomics-and-lipidomics 